\title{
Extinction of operant behavior: An analysis based on foraging considerations
}

\author{
ROGER L. MELLGREN \\ University of Texas, Arlington, Texas \\ and \\ TIMOTHY F. ELSMORE \\ Walter Reed Army Institute of Research, Washington, D.C.
}

\begin{abstract}
In two experiments, the frequency of food reinforcement provided by variable interval (VI) schedules prior to extinction was varied. In the first experiment, two-component multiple schedules resulted in a greater number of responses in extinction in the presence of the stimulus previously associated with the richer of the two component schedules than that previously associated with the leaner schedule. In the second experiment, different groups of animals were trained on different VI schedules. Responding in extinction was analyzed into bouts of responding showing that the number of response bouts increased and the number of responses per bout decreased with decreasing frequency of reinforcement during training. These data are compatible with an analysis of operant behavior based on an analogy to processes that presumably occur in naturalistic foraging situations. According to this analogy, behavior associated with search for a food source (i.e., number of response bouts) and that of procurement of food from a source (i.e., responses per bout) represent aspects of behavior that are differentially strengthened by different VI schedules. Extinction serves to reveal this differential strengthening.
\end{abstract}

It has been suggested that performance in the Skinner box is a useful analogue to aspects of foraging as it occurs in nature (e.g., Baum, 1983; Collier \& RoveeCollier, 1981; Fantino \& Abarca, 1985; Kamil \& Sargent, 1981; Killeen, Smith, \& Hanson, 1981; Lea, 1979, 1981; Mellgren \& Olson, 1983; Shettleworth, 1988, 1989). It may be argued that an operant chamber is analogous to a patch, or clump of food, that occurs in a natural environment and the problem the forager/subject faces is primarily one of procurement of the food available in the patch. An operant chamber is a very strange environment, however, because there is only one patch available and the forager is confined to a single location.

Foraging also involves several factors other than procurement of food from a patch. Among these other factors, search for and location of a patch are of obvious importance. Some of the maze procedures used in the laboratories of experimental psychologists may be analogous to searching and locating a patch in the natural en-

In conducting the research described in this report, the investigators adhered to the Guide for the Care and Use of Laboratory Animals, as promulgated by the Committee on Care and Use of Laboratory Animals of the Institute of Laboratory Animal Resources, National Research Council. The views of the authors do not purport to reflect the position of the Department of the Army or the Department of Defense (Para. 4-3, AR 360-5). Address correspondence to either Roger L. Mellgren, Department of Psychology, University of Texas at Arlington, Box 19528, Arlington, TX 76019, or Timothy F. Elsmore, Department of Medical Neurosciences, Walter Reed Army Institute of Research, Washington, DC 20307. vironment (Batson, Best, Phillips, Patel, \& Gilleland, \& 1986; Ilersich, Mazmanian, \& Roberts, 1988; Mellgren \& Olson, 1983; Roberts \& Iersich, 1989). "Search," as defined in the laboratory context, is a behavior that brings the subject into contact with a food source, or patch, and "contact with a patch" means being close enough to the response manipulandum in a Skinner box to be able to press it. Obviously, search involves movement through space, but contact with a patch (procurement) does not require movement through space. Rather, it involves repeated motor actions in contact with (or in close proximity to) the response manipulandum. Therefore, we argue that as long as the rat maintains contact and/or close proximity to the response manipulandum in the operant box it is "procuring." When the rat breaks contact with the manipulandum, it recontacts the lever only when it reinstates a "searching" process. As long as the schedule of reinforcement remains in force, the subject will tend to stay in contact with the patch and be procuring. Essentially, the schedule of reinforcement dominates and controls the subject's behavior. The domination of the schedule is probably enhanced by the fact that standard schedules used in operant studies maintain a constant payoff over time, but in nature most patches deplete with continued procurement and therefore result in the subject's breaking contact with the patch. The extinction schedule, however, is a condition in which contact with the patch will not be continuous because it represents a patch in which total depletion has occurred. Thus, the extinction schedule is analogous to a patch that has been totally depleted of resources. 
In laboratory studies of animal learning, extinction has often been used as a method for assessing the nature of learning that has preceded the extinction phase. Hearst (1986), for example, argues that extinction is a useful procedure for revealing those things that were learned during acquisition, but not evident in the behavior of the subject during the acquisition phase. This point has also been made by researchers interested in the partial-reinforcement effect (Amsel, 1967; Capaldi, 1967), a phenomenon that depends on transfer from different acquisition schedules to an extinction schedule for its definition.

Although there is a voluminous literature using extinction performance in runways as the main dependent variable to evaluate the effects of different schedules of reinforcement, very few of the data have come from the operant-box situation (e.g., Robbins, 1971). Exceptions to this generalization involve attempts to use operant methods in a manner analogous to runway studies (e.g., Bitgood \& Platt, 1971; Haddad, Walkenbach, \& Goeddel, 1980; Overmann \& Denny, 1974) or studies done in the operant box in which there was no analogy to runway procedures, but the results were interpreted with respect to theories developed using runway data as their base (e.g., Pavlik \& Carlton, 1965; Pavlik \& Collier, 1973). In addition, there have been some operant studies by Nevin and his colleagues (Nevin, 1974, 1979; Nevin, Mandell, \& Atak, 1983; Nevin, Mandell, \& Yarensky, 1981) that were independent of both method and theory developed in the runway, and Nevin (1988) has addressed some of the apparent contradictions between operant and runway data with respect to the partial-reinforcement effect. Other relevant operant studies are primarily of historical interest (e.g., Jenkins, McFann, \& Clayton, 1950; Perin, 1942; Skinner, 1938; Williams, 1938).

In the experiments described in this article, extinction of responding following variable interval schedules of food reinforcement was viewed as reflecting processes analogous to those that might occur in a foraging situation. In particular, the processes we hypothesized to be operating are those of search and procurement. "Search" was operationally defined as a period of noncontact with the response manipulandum terminated by contact with the manipulandum. We do not mean that the subject is exclusively engaged in searching behavior when not in contact with the response lever, but that the recontact with the lever after a period of absence of contact reflects the occurrence of searching. "Procurement" was operationally defined as sustained contact with the manipulandum. According to our analysis, the proper method for evaluating the extinction of operant behavior is with respect to these two processes. Timberlake and Lucas (1989) suggest a similar analysis based on the proximity of behavior relative to consumption of food. In their terminology, the "general search mode" is roughly what we call "search" and "focal search/food handling"' is what we call "procurement." Timberlake and Lucas suggest that the partial reinforcement effect will be evident for behaviors of the general search mode, but the opposite will occur for focal search/food handling behaviors $(1989$, p. 269).
Previous research on the effect of relative density of scheduled reinforcers on resistance to extinction is consistent with the conclusion that search (general search) and procurement (focal search) represent different processes. The resistance to extinction shown by subjects in runways increases as percentage of reinforcement decreases (Robbins, 1971). In the operant situation, the subject is already in the potential food source and the most critical process is that of procurement by repeated contact with the response manipulandum. Studies that vary the density of scheduled reinforcers on both interval and ratio schedules in the operant box show that resistance to extinction increases as the density of reinforcement increases: the opposite result of the runway studies (Nevin, 1979).

We take the differences between runway and operant studies to reflect the relative importance in each procedure of search and procurement processes, that is, the components of foraging behavior as it occurs in nature. On the other hand, there are several differences between the two procedures that might also contribute to differences in results. The runway data are based on betweensubjects comparisons, but the operant data are based on within-subject comparisons. We consider both types of comparisons in the present studies. The dependent variable in runway studies is the time to respond (or its reciprocal, speed), but rate of response is used in operant studies. The experiments in this report were designed and analyzed in order to test the hypothesis that search and procurement are differentially affected by schedules of reinforcement as measured during extinction, with the objective of reconciling the apparent differences between runway and operant data. Experiment 1 utilizes multiple schedules for within-subject comparisons, and Experiment 2 uses between-subjects schedule comparisons, analogous to the typical runway methodology.

\section{EXPERIMENT 1}

Previous work on "response strength" (Nevin, 1979) has documented that following multiple variable interval (mult VI) schedules in which different VIs are associated with different cues, there is greater response strength in extinction in the component of the schedule that formerly was associated with the richer schedule. In order to maintain continuity with this previous work, Experiment 1 used VI 18-, 56-, and 180-sec schedules, with one group of rats receiving a mult VI18 VI56 schedule and the other group receiving a mult VI56 VI 180 schedule. Both groups were then given extinction to both components, mult (ext, ext), with the expectation that more responding would occur in the formerly rich component of the multiple schedule.

\section{Method}

Subjects. Twelve experimentally naive male albino SpragueDawley rats approximately 90 days old were randomly divided into two groups of 6 each. They were maintained on $12 \mathrm{~g}$ of Purina lab chow per day in addition to the approximately $4.5 \mathrm{~g}$ of food pellets 
they earned in the experiment. All rats were fed at the same time, approximately $15 \mathrm{~min}$ after the last subject finished the session. Water was available continuously in the home cage, and the rats were weighed daily.

Apparatus. Twelve operant conditioning chambers (Colbourn Instruments) were used. Each chamber was equipped with two response levers located $6.5 \mathrm{~cm}$ from the floor of the chamber and separated by the food-delivery tray. Small pilot lights covered by white glass jewels centered $3.5 \mathrm{~cm}$ above each lever served as discriminative stimuli for the multiple schedules. The chambers were housed in sound-attenuating shells, and ventilation and masking noise were provided by exhaust fans mounted on the shells. Control of the apparatus and recording of responses was accomplished by a PDP/8e computer running the SUPERSKED software system (Snapper, Kadden, \& Inglis, 1982).

Procedure. The rats were given $12 \mathrm{~g}$ of food per day for 8 days prior to the start of the experiment. They were then magazine trained by delivering food pellets on a variable time (VT) 56-sec schedule, with both response levers programmed to deliver pellets on a continuous reinforcement (CRF) schedule. Throughout both experiments, food-pellet delivery was accompanied by a 1-sec illumination of the food-delivery tray. A total of 100 pellets was given on the first session, either via the VT schedule or a combination of the VT schedule and leverpresses. For those subjects that did not press the lever during the first pretraining session, the experimenter shaped leverpressing on the next session. On the third pretraining session, a multiple CRF, CRF schedule was in effect. On this schedule, the light over the left or right lever was randomly chosen and illuminated. A press on the lever under the light resulted in delivery of a food pellet and another random selection of the operative lever. A total of 100 pellets were obtained by the subject in this session.

Half of the subjects received a multiple variable interval 18-sec, variable interval 56-sec schedule (mult VI 18 VI 56), and the other half received a multiple variable interval $56-\mathrm{sec}$, variable interval 180 -sec schedule (mult VI 56 VI 180), for seven sessions. Intervals of the schedules were selected according to the method of Fleshler and Hoffman (1962). Ilumination of the light above a lever was the signal that the lever was operative, and left and right lever lights were counterbalanced so that the richer schedule was on the left for half the subjects and on the right for the other half. Presses on the unlighted lever side were recorded, but had no programmed consequences. Each component was presented for $90 \mathrm{sec}$, after which the computer randomly sampled to determine which component would occur next. All lights were off and responses had no effect for $1 \mathrm{sec}$ between components. To equate the number of pellets delivered on each lever, the ratio of sampling a particular component was 3 to 1 in favor of the less rich component (the VI56 for the mult VI 18 VI 56 group and the VI 180 for the other group). For the group on the mult VI 18 VI56 schedule, a total of 42 components was presented, and for the group on the mult VI56 VI 180 schedule, a total of 132 components was presented. This arrangement would result in the subjects receiving approximately 50 pellets per component in each session. In actuality, the subjects earned somewhat fewer pellets than they theoretically might have earned (usually around 43 to 47 per component).

After seven sessions of acquisition training, extinction testing was done. The extinction session began with 8 presentations of reinforced components, 4 of the richer and 4 of the leaner, followed by 80 extinction presentations, 40 of each component, with the pellet dispenser turned off. Components lasted for $90 \mathrm{sec}$, and their order was randomly determined with the restriction that exactly 40 presentations of each component occurred in the session.

\section{Results}

The degree of stimulus control exhibited by the multiple schedule was evaluated in two ways. One index of discriminative control is the proportion of total responses on the correct lever. On Day 1 of acquisition, the mult VI 18 VI56 group showed an accuracy level of 95.4 on the VI 18 component and 85.4 on the VI56 component, and they maintained this level through the course of acquisition, ending with $\mathbf{9 6 . 8}$ and 82.0 , respectively, on the seventh session. Discriminative control remained high in extinction at 94.2 and 90.8 , respectively. The mult V156 VI 180 group did show improvement on this index, starting at 87.7 and 77.8 for the VI56 and VI 180 components, and ending at 94.7 and 96.4 , respectively, on the seventh session. Again, discriminative control remained high in extinction, 94.8 and 99.4 , respectively.

Another index of discriminative performance is the relative response rate in each component of the schedule, that is, the degree to which the components of the multiple schedules controlled different rates of response. These data showed that the main change in responding across the course of acquisition was an increase in rate in the VI 18 component for the mult VI 18 VI56 condition, whereas responding remained constant in the other components. Comparison of the VI56 component between the two groups showed no difference in response rate, that is, a failure to observe a "nonspecific contrast effect" (cf. Dunham, 1968).

The density of schedule varied both within subjects and between subjects. We refer to the rich component as the VI 18 component for the mult VI 18 VI56 condition and the VI56 component for the mult VI56 VI 180 condition. The two conditions, mult VI 18 VI56 and mult VI56 VI 180, differed in the overall richness of schedules (the combined rate of pellet delivery provided by the multiple schedule), and the effects of this difference are referred to as "group" effects.

The analysis of response rate in extinction included rich versus lean schedules (within subject) as one variable and mult VI 18 VI56 versus mult VI56 VI 180 as the other (between subjects). All statistical analyses that are referred to as significant are at the .05 level of significance or better. As would be expected from Nevin's (1974) previous findings, the richer schedules resulted in higher response rates in extinction than did leaner schedules $[F(1,10)=$ 18.52]. The mult VI 18 VI56 group had a higher response rate in extinction than did the mult VI56 VI 180 group $[F(1,10)=6.59]$. The interaction of component richness with groups $[F(1,10)=6.43]$ is accounted for by the relatively bigger difference in rate between the rich and lean components for the mult VI 18 VI56 group as compared with the mult VI56 VI 180 group. Of course, this pattern of extinction results is similar to the differences that existed at the end of acquisition as shown in Table 1 . Therefore, the extinction rates were transformed by dividing the extinction rate by the rate of response on the last day of acquisition (Anderson, 1963).

The transformed extinction response rates showed no significant difference due to the richness of the component schedules or the group effect, but did show a significant interaction between the two variables $[F(1,10)=$ 9.17]. The transformed extinction rates were much higher 
Table 1

Behavioral Measures for Mult VI 18 VI 56 or Mult VI56 VI 180 Training

\begin{tabular}{lccccc}
\hline & \multicolumn{4}{c}{ Schedule } \\
\cline { 2 - 3 } \multicolumn{1}{c}{ Dependent Variable } & \multicolumn{2}{c}{ Mult VI 18 VI56 } & & Mult VI56 VI 180 \\
\cline { 2 - 3 } \cline { 6 - 7 } \multicolumn{1}{c}{ Rich } & Lean & & Rich & Lean \\
\hline Acquisition rate (in minutes) & 57.5 & 26.6 & & 22.7 & 9.9 \\
Extinction rate (in minutes) & 15.0 & 3.7 & & 5.4 & 2.5 \\
Extinction rate (transformation) & .26 & .14 & & .24 & .25 \\
Latency (in seconds) & 46.51 & 52.92 & & 37.64 & 38.00 \\
Frequency of nonresponse & 14.33 & 18.17 & & 10.16 & 11.67 \\
\hline
\end{tabular}

in the richer component for the mult VI 18 VI56 condition, but there were no differences between the components of the mult VI56 VI 180 condition, as shown in Table 1 . The time course of extinction was considered by plotting the transformed extinction rates (using the first block of extinction as the denominator for the transformation) in blocks of 5 and 10 components. The slope of the rates failed to reveal systematic differences between rich and lean components, in contrast to other results (Nevin, 1988).

In discrete trial, runway extinction studies, the usual dependent variable is the latency, or time to respond (or its transformation, speed of response). Therefore, we analyzed the time to the first leverpress response after a component light was presented, assuming it to be analogous to latency in the runway. Failure to respond for the 90 sec presentation of one component was given the value of 90 . Figure 1 shows the mean latencies (in seconds) across blocks of 10 component presentations.

An analysis of variance showed that the mult VI 18 VI56 group had significantly longer response latencies than did the mult VI56 VI 180 group $[F(1,10)=7.53]$. Schedule richness and the interaction of groups with schedule richness were not significant sources of variance $[F(1,10)=$ 1.00 and 0.83 . There was a significant effect due to

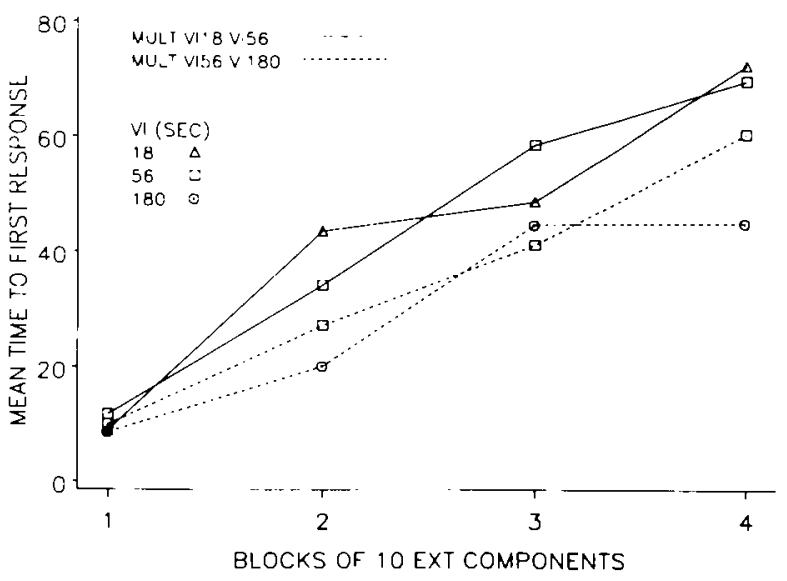

Figure 1. Mean time to first response following presentation of the cue light starting a component across the $2 \mathrm{~h}$ of extinction in Experiment 1. Nonresponse components were assigned a value of 90 . blocks $[F(3,30)=3.87]$. Overall, the groups started extinction at the same level and diverged across blocks.

The number of components in which no response occurred showed a pattern that would be expected from the time data. There were more components without a response for the mult VI 18 VI56 group than for the mult VI56 VI 180 group $[F(1,10)=5.65]$, but no difference due to schedule richness or the interaction of groups with schedule richness $[F(1,10)=0.10$ and 0.45$]$. Table 1 shows both the latency averaged across extinction and the nonresponse data.

The conclusion about the effect of the richness, or density, of multiple schedules on the persistence of behavior in extinction is that, "it depends." It depends on what measure of behavior is used to assess resistance to extinction and on whether the comparison is made between components but within subjects, or just between subjects. Relative rate of response (adjusted for different rates in acquisition) shows more resistance to extinction for the denser schedule in the mult VI 18 VI56 group, but no between-groups difference. However, the absolute rate of response is higher following denser schedules both for a within-subject, between-components comparison and for a between-groups comparison. The response time and the tendency to make at least one response in a component suggest the opposite conclusion. There were shorter response times and a higher likelihood of making at least one response for less dense schedules between groups of subjects, supporting the conclusion that the less dense the schedule of reinforcement, the greater the resistance to extinction. Although these conclusions are seemingly contradictory, they are consistent with existing literature when viewed from the perspective of the foraging analogy described in the introduction of this article. This analogy claims that there are two processes controlling operant behavior. One is the tendency to "search," which we operationalize as the tendency to contact physically the response lever after a period away from it, and the other is the tendency to "procure," which we operationalize as the tendency to continue leverpressing once contact with the lever has occurred. The search process is usually isolated in the straight runway experiment, in which the subject is introduced into the situation by the experimenter, allowed to approach a potential food source, and then removed by the experimenter. Generally, the runway data are consistent in showing that the tendency to approach 
during extinction increases as density of scheduled food decreases. In the present situation, the tendency to approach is measured by the response time following presentation of the cue light. The between-group extinction comparison in this operant-box experiment is therefore consistent with between-groups runway findings in showing a shorter response time (and greater likelihood of response) with lower density of food in acquisition.

The tendency to persist in leverpressing once responding has been initiated is called the procurement process. If only overall number or rate of responses is considered in analyzing the data, then the procurement process tends to predominate and produce results that favor the hypothesis that resistance to extinction is greater following denser schedules of reinforcement, as was found. This finding is true even though we have not factored out the hypothesized "search time" at the beginning of each component.

\section{EXPERIMENT 2}

According to the foraging analogy to the operant situation, the use of a multiple schedule facilitates a particular pattern of behavior. We hypothesized that leverpressing is analogous to the procurement of food from a patch. Cessation of leverpressing and moving away from the lever, followed by the reinitiation of contact, is analogous to searching for a food source in the natural situation. In a multiple schedule, the termination of one component and initiation of another are not under the control of the subject, but are under the control of the experimenter, a situation not strictly analogous to the foraging situation. In this experiment, different groups of subjects were given simple experience with one value of a VI schedule followed by extinction. On the basis of the results of Experiment 1 , we hypothesized that there would be a direct relationship between the density of the programmed reinforcer in acquisition and the persistence of responding, once responding was initiated, during extinction. It is important to note that it is only once the subject has initiated leverpressing that the number of responses is predicted to be directly related to the scheduled density of reinforcement. The foraging analogy suggests that tendency to initiate contact with the patch (leverpress) is inversely related to previous schedule density. This hypothesis is consistent with the runway literature showing that persistence of responding increases as density of reinforcement decreases. Therefore, we hypothesize two opposite effects on the persistence of behavior as a function of the density of reinforcement. When measured as the tendency to leverpress (referred to as starting a "bout" of responding), persistence should increase as density decreases. When measured as the tendency to continue leverpressing once a bout has been initiated, persistence should decrease as density decreases.

\section{Method}

Subjects. Naive rats like those used in Experiment 1 were used in this experiment. Two replications of 12 rats were run, with 4 subjects per group per replication.
Apparatus. The same chambers and control apparatus were used in this experiment as were used in Experiment 1. Only the right lever was functional, and illumination was provided by the left light.

Procedure. The subjects were given $12 \mathrm{~g}$ of food per day for 8 days and then were placed individually in the operant chambers and given food pellets on a VT56 schedule and CRF for leverpresses until 100 pellets had been received. A second day of these conditions was given to all subjects, and the rats that had not pressed the lever during the first session were given shaping by the experimenter. By the end of the second session, all subjects had learned to press the lever. The subjects were randomly distributed into one of three groups, VI 18, VI56, or VI 180, and received the appropriate schedule for the next 8 sessions. The computer controlled the duration of a session so that once the subject had eamed 100 pellets, the light inside the box went out, the lever became inoperative, and a signal was delivered to the experimenter indicating that a subject was ready to be removed from the apparatus. This procecture resulted in average session durations of 32-38 min for the VI 18 condition, 102-110 $\mathrm{min}$ for the VIS6 condition, and 320-330 min for the VI 180 condition. All subjects were given the daily food ration in the home cage approximately $15 \mathrm{~min}$ after the last subject in the VI 180 condition had finished.

The extinction session was conducted the day after the eighth acquisition session. It began with the subject's earning 10 pellets on the appropriate schedule and continued for $2 \mathrm{~h}$ after the 10th pellet was received. During extinction, each response and its time of $\alpha$ currence were recorded by the computer for later analysis.

\section{Results}

To provide a general picture of extinction, analyses of variance were done on the response rate during extinction and the transformed measure of extinction, that is, extinction rate divided by acquisition rate. The baseline response rate as measured by the last session before extinction varied as a function of schedule $[F(2,18)=$ 22.18], with higher density of available food resulting in higher response rates. Although extinction response rate increased with increasing acquisition schedule density, the differences were not statistically significant $[F(2,18)=$ 2.68] (see Table 2). The derived measure of extinction, response rate in extinction divided by response rate in acquisition baseline, increased significantly as previous schedule of reinforcement decreased in density $[F(2,18)$ $=24.61]$.

The pattern of results shown in Table 2 and the corresponding analyses do not provide a definitive insight into between-group extinction behavior following different values of variable interval schedules. On the one hand, response rate was greater with more densely scheduled reinforcers, but the differences failed to reach statistical significance. The fact that groups differed in their baseline rates at the start of extinction further complicates the

Table 2

Behavioral Measures as a Function of Schedule of Reinforcement

\begin{tabular}{lrrr}
\hline & \multicolumn{3}{c}{ Schedule } \\
\cline { 2 - 3 } Dependent Variable & VI18 & VI56 & VI 180 \\
\hline Baseline rate & 52.56 & 27.73 & 8.46 \\
Extinction rate & 9.02 & 7.17 & 3.29 \\
Extinction/baseline & .15 & .29 & .43 \\
\hline
\end{tabular}


interpretation of the data. Anderson (1963), for example, advocates the use of a transformation on extinction data when there are differences in performance levels at the end of acquisition. When this transformation is performed on the present data, a totally different picture emerges compared with the analysis of untransformed extinction response rates. The transformed measure (extinction rate relative to baseline) indicates that the lower density schedules produce greater persistence of responding than do the higher density schedules. The fact that the baseline response rates are so different encourages caution in interpreting differences in extinction based on a transformation of this sort. The observed differences in the transformed measure of extinction may be more a function of the differences in acquisition rates than a revelation of anything about extinction. Therefore, we turn to a more detailed evaluation of the extinction of responding for a clearer picture of the controlling processes.

The foraging analogy of operant behavior suggests that extinction of operant responding involves two processes, procurement and reinstatement of procurement following a pause in responding (search). This analysis suggests that operant responding, as defined by leverpressing, occurs in discrete bouts. The tendency to continue responding within a bout is one measure of the persistence of behavior. The tendency to engage in a bout of responding once leverpressing has been terminated is a different measure of the persistence of behavior. Therefore, the extinction data were analyzed for the occurrence of bouts of responding. The definition of when a bout had terminated was made by measuring the amount of time between successive leverpresses. When a pause (an interresponse time, IRT) between responses $n$ and $n+1$ of criterion duration or longer occurred, the computer treated the $n$th response as a member of bout number $x$ and response $n+1$ as a member of bout number $x+1$. There is no a priori way of deciding what the criterion length of the IRT should be; therefore, a range of criteria was used to define bouts. Analyzing the data in this way resulted in two dependent variables: number of bouts and number of responses within bouts for each bout criterion.

The less dense the acquisition schedule, the greater the number of bouts. This relationship held regardless of the IRT used to define the bout criterion. The bout-termination criteria were $5,10,20$, and $40 \mathrm{sec}$. The analyses of variance showed significant differences due to schedule for all criteria $[F(2,18)=8.96,20.93,21.70$, and 7.43]. Figure 2 shows the number of response bouts for the different bout-termination criteria.

The denser the acquistion schedule, the greater the number of responses per bout. This relationship held regardless of the IRT used to define bouts. The analyses of variance showed significant differences due to schedule for all criteria except $40 \mathrm{sec}[F(2,18)=9.23,6.40,4.71$, and 2.75]. Figure 3 shows responses per bout for the different bout-termination criteria.

The summary of performance of the subjects seen in Figures 2 and 3 is also seen in the data of individual sub- jects. Figure 4 shows all of the cumulative records from individual subjects in each of the VI groups. Rather than plotting raw responses in which the differences in response rates would make it difficult to compare subjects and VI conditions, the cumulative records are presented as cumulative percentages of total responses in the extinction session. The pattern of pausing and then emitting a long bout of procurement responding in the VI 18 subjects gives a different appearance than the relatively smoother and less steep cumulative records of the VI 180 subjects. The VI56 schedule was intermediate to the other two.

\section{Discussion}

The results of Experiment 2, when analyzed using standard dependent variables such as response rate, number of responses, or transformed response rate based on acquisition rate, are equivocal. Perhaps the reason there is little published data on extinction following simple acqui-

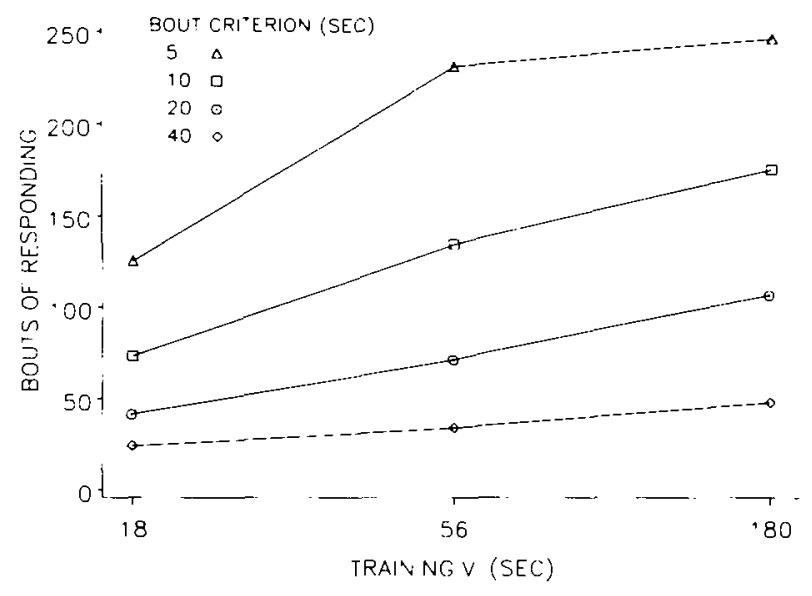

Figure 2. Mean number of bouts of responding in Experiment 2 for the three variable interval conditions, using four bout-termination criteria.

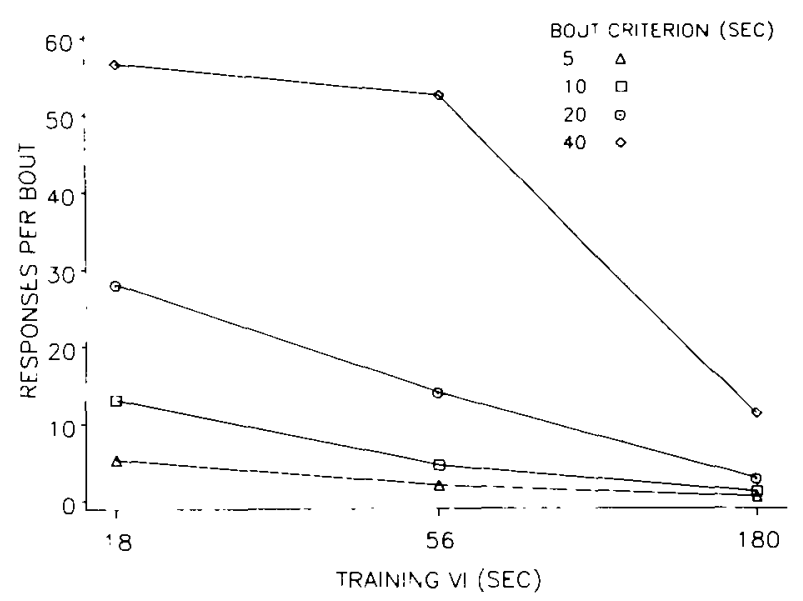

Figure 3. Mean number of responses per bout in Experiment 2 for the three variable interval conditions, using four bout-termination criteria. 


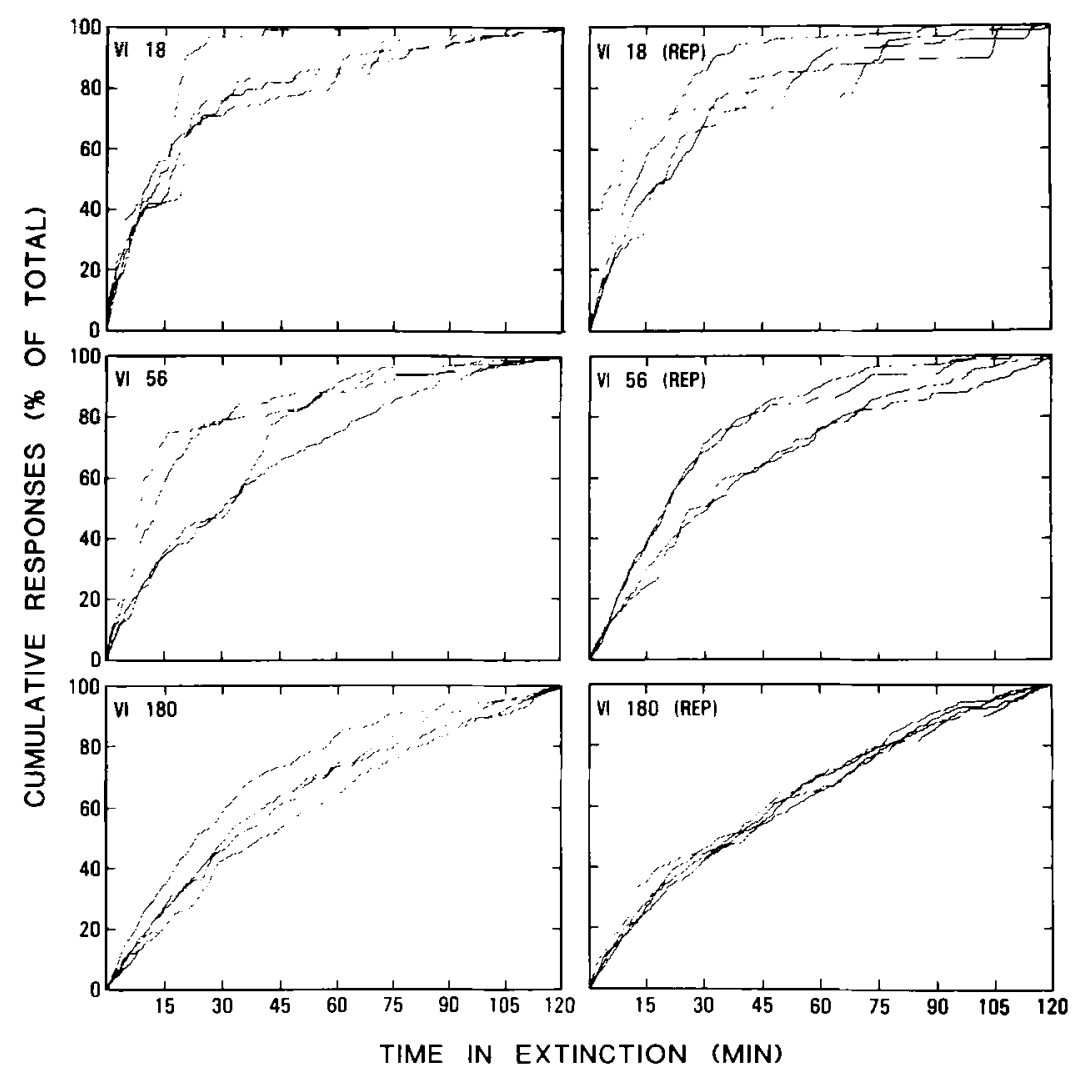

Figure 4. Cumulative records of the percentage of total responses in extinction for each subject in Experiment 2. The len panels are for the first squad of subjects, and the right panels are for the second, or replication, squad.

sition on a schedule is because the results are not definitive when the standard dependent variables are analyzed.

Dividing extinction into two components, search and procurement, implies that the appropriate dependent variables are the "nonstandard" ones, bouts of responding and responding within bouts. Evaluating bouts and responses within bouts results in opposite conclusions regarding the resistance to extinction following different density schedules. Interestingly, this pattern of results is consistent with the existing literature if the analogy between search and the runway procedure and between procurement and the leverpressing procedure on multiple schedules is maintained. In runway investigations, increased resistance to extinction is associated with decreasing density of reinforcement, as was found with respect to the number-of-bouts dependent variable in this experiment. In operant investigations using multiple schedules of reinforcement, increased resistance to extinction is associated with increasing density of reinforcement, as was found when the number of responses in a bout was the dependent variable.

\section{GENERAL DISCUSSION}

We have attempted to show that resistance to extinction, and more generally, persistence of behavior, reflects the operation of processes that govern foraging behavior in the natural environment. For an omnivore such as the rat, foraging may take many forms. At a minimum, food must be located and, once located, must be harvested or procured. The degree to which laboratory preparations are consistent with the naturally occurring foraging behaviors of the subject may determine the generality of the results (Shettleworth, 1975). It is our contention that the laboratory preparations commonly used to study instrumental learning are closely related to two of the fundamental problems of foraging: search and location of a food source, and harvesting or procurement of food from the source. Runway studies are argued to be analogous to the search process as it occurs in foraging. Once a food source has been located, there is often the additional problem of obtaining the available food. "Handling," as it is often called in the foraging literature, or "procurement," as we call it, has its analogy in the Skinner box apparatus. Under most of the standard procedures used in laboratory studies, the focus is on one or the other of the processes related to foraging. In runway studies (and maze studies in general), when food is found it is immediately available in a food cup and comes packaged in soft pellets so that the rat does not even touch the food with its paws in consuming it. In Skinner box studies, the subject is placed into the box and obtains food by repeatedly 
contacting the response manipulandum in a fixed location. Thus, there is no influence of search processes as long as the subject is under the control of the procurement requirement dictated by the schedule of reinforcement and remains in contact with the manipulandum.

The argument that runways focus upon search processes while Skinner boxes focus upon procurement processes does not mean that each type of apparatus must necessarily exclude the other process. The animal comes equipped to engage in both search and procurement functions since they are both parts of the feeding behavior system (Timberlake and Lucas, 1989), and when the experimental situation is structured in a way that requires both processes, the animal is capable of engaging in both. For example, Mellgren and Olson (1983) had rats run down a runway to a "goal box" partially filled with sand. The rats had to dig in the sand to find buried food. The running to the sand box clearly fits the analogy to search, and the digging for food clearly fits the analogy to procurement. In extinction, the persistence of running to the sand box was greater if food had been found only half the time during acquisition compared with all the time. Digging, on the other hand, was more persistent if food had been present on every trial in acquisition than if it had been present on only half the trials. These results are consistent with those reported in this article in showing that resistance to extinction may depend on the function of the behavior, that is, search or procurement.

Another important dimension for understanding extinction effects is the nature of the comparison (betweensubject or within-subject paradigms) and the dependent variable (time to respond or number of responses) used to assess relative resistance to extinction. Response latency (or its transformation, response speed) seems relatively insensitive when measured using a within-subject dependent variable, but relatively sensitive when used in conjunction with a between-subjects dependent variable. In Experiment 1, the time-to-respond data showed no differences between the VI 18 and VI56 components in the mult VI 18 VI56 group and no differences between the VI56 and VI 180 components in the mult VI56 VI 180 group. This insensitivity in the time-to-first-response measure occurred despite clear discrimination between the components in the number of responses made to each. Interestingly, the between-subjects comparison for the same measure was significant. The mult VI18 VI56 group, although making many more total responses in extinction, was significantly slower to make the first response in either component than the mult VI56 VI 180 group. Of course, the usual dependent variable in runway studies is time to run from the start box to the goal box, and the usual comparison is a between-subjects one. It is interesting that the parallel dependent variable and experimental design produced parallel results between the runway and the Skinner box in this instance.

The time-to-respond data from Experiment 1 are consistent with the idea that lower density VIs should result in more bouts of responding during extinction in Experi- ment 2. There was, of course, a major difference between Experiments 1 and 2 since the opportunity to engage in a bout of responding was enabled by the experimenter in the multiple-schedule procedure of Experiment 1, but was under the subject's control in Experiment 2. The withinsubjects difference in total responses in each component of the multiple schedule comes about because of the "procurement" difference between schedules. Once responding begins in the denser component, more of it occurs there than in the less dense schedule. When both components are presented an equal number of times in extinction, the "procurement responding" results in a greater total number of responses in the denser schedule. Despite these differences in total responses, there were no differences in initiation of responding within groups between the components of the multiple schedules. This pattern of results lends further support to the hypothesis that search and procurement represent separate processes that are not similarly affected by a given outcome and illustrates the usefulness of this analysis in understanding appetitive behavior.

\section{REFERENCES}

AMSEL, A. (1967). Partial reinforcement effects on vigor and persistence. In K. W. Spence \& J. T. Spence (Eds.), The psychology of learning and motivation (Vol. 1, pp. 1-65). New York: Academic Press.

Anderson, N. H. (1963). Comparison of different populations: Resistance to extinction and transfer. Psychological Review, 70, 162-179.

Batson, J. D., Best, M. R., Philups, D. L., Patel, H., \& Gilleland, K. R. (1986). Foraging on the radial-arm maze: Effects of altering the reward at a target location. Animal Learning \& Behavior, 14, 241-248.

BAUM, W. M. (1983). Studying foraging in the psychological laboratory. In R. L. Mellgren (Ed.), Animal cognition and behavior (pp. 253283). Amsterdam: North-Holland.

Bitgood, S. C., Platt, J. R. (1971). A discrete-trials PREE in an operant situation. Psychonomic Science, 23, 17-19.

CAPALDI, E. J. (1967). A sequential hypothesis of instrumental learning. In K. W. Spence \& J. T. Spence (Eds.), The psychology of learning and motivation (Vol. 1, pp. 67-157). New York: Academic Press.

Collier, G. H., \& Rovee-Coluser, C. K. (1981). A comparative analysis of optimal foraging behavior: Laboratory simulation. In A. C. Kamil \& T. D. Sargent (Eds.), Foraging behavior: Ecological, ethological, and psychological approaches (pp. 39-76). New York: Garland.

Dunham, P. J. (1968). Contrasted conditions of reinforcement: A selective critique. Psychological Bulletin, 69, 295-315.

Fantino, E., ABARCa, N. (1985). Choice, optimal foraging, and the delay-reduction hypothesis. Behavioral \& Brain Sciences, 8, 315-362.

Fleshler, M., Hoffman, H. S. (1962). A progression for generating variable interval schedules. Journal of the Experimental Analysis of Behavior, 5, 529-530.

Haddad, N. F., Walkenbach, J., \& Goeddel, P. S. (1980). Sequential effects on rats' lever-pressing and pigeons' key-pecking. American Journal of Psychology, 93, 41-51.

HEARst, E. (1986). Extinction reveals stimulus control: Latent learning of feature-negative discriminations in pigeons. Journal of Experimental Psychology: Animal Behavior Processes, 13, 52-64.

Ilersich, T. J., MaZmanian, D. S., \& Roberts, W. A. (1988). Foraging for covered and uncovered food on a radial maze. Animal Learning \& Behavior, 16, 388-394.

Jenkins, W. O., MCFANN, H., Clayton, F. L. (1950). A methodological study of extinction following aperiodic and continuous rein- 
forcement. Joumal of Comparative \& Physiological Psychology, 43, 155-167.

KAMIL, A. C., SARGENT, T. D. (Eds.) (1981). Foraging behavior: Ecological, ethological and psychological approaches. New York: Garland.

Killeen, P. R., Smith, J. P., \& Hanson, S. J. (1981). Central place foraging in Rattus norvegicus. Animal Behavior, 29, 64-70.

LEA, S. E. G. (1979). Foraging and reinforcement schedules in the pigeon: Optimal and nonoptimal aspects of choice. Animal Behavior. 27, 875-886

LEA, S. E. G. (1981). Correlation and contiguity in foraging behavior. In P. Harzem \& M. D. Zeiler (Eds.), Advances in analysis of behavior: Vol. 2. Predictability, correlation, and contiguity (pp. 355406). Chichester, England: Wiley.

Mellgren, R. L., \& Olson, M. W. (1983). Mazes, Skinner boxes, and feeding behavior. In R. L. Mellgren (Ed.), Animal cognition and behavior (pp. 223-252). Amsterdam: North-Holland.

NEVIN, J. A. (1974). Response strength in multiple schedules. Joumal of the Experimental Analysis of Behavior, 39, 49-59.

NEVIN, J. A. (1979). Reinforcement schedules and response strength. In M. D. Zeiler \& P. Harzem (Eds.), Reinforcement and the organization of behavior (pp. 117-158). New York: Wiley.

Nevin, J. A. (1988). Behavioral momentum and the partial reinforcement effect. Psychological Bulletin, 103, 44-56.

Nevin, J. A., MANDell, C., Atak, J. R. (1983). The analysis of behavioral momentum. Joumal of the Experimental Analysis of Behavior, 39. 49-59.

Nevin, J. A.. MANdell, C., Yarensky, P. (1981). Response rate and resistance to change in chained schedules. Joumal of Experimental Psychology: Animal Behavior Processes, 7, 278-294.

Overmann, S. R., Denny, M. R. (1974). The free-operant partial reinforcement effect: A discrimination analysis. Leaming \& Motivation, 5, 248-257.

PAvlik, W. B., CARlton, P. C. (1965). A reversed partial reinforcement effect. Joumal of Experimental Psychology, 70, 417-425.

PAvlik, W. B., Coluer, A. C. (1973). Reinforcer magnitude effects on a within-subjects reversed PRE. Bulletin of the Psychonomic Society, 2, 233-234.
Perin, C. T. (1942). Behavior potential as a joint function of the amount of training and degree of hunger at the time of extinction. Joumal of Experimental Psychology, 30, 93-113.

Robins, D. (1971). Partial reinforcement: A selective review of the alleyway literature since 1960. Psychological Bulletin, 76, 415-431.

RoBerTs, W. A., ILersich, T. J. (1989). Foraging on the radial maze: The role of travel time, food accessibility, and the predictability of food location. Joumal of Experimental Psychology: Animal Behavior Processes, 15, 274-285.

SHETTLEWORTH, S. J. (1975). Reinforcement and the organization of behavior in golden hamsters: Hunger, environment, and food reinforcement. Journal of Experimental Psychology: Animal Behavior Processes, 104, 56-87.

SHETTLEWORTH, S. J. (1988). Foraging as operant behavior and operant behavior as foraging: What have we learned? In G. Bower (Ed.), The psychology of leaming and motivation: Advances in research and theory (Vol. 22, pp. 1-32). New York: Academic Press.

Shettleworth, S. J. (1989). Animals foraging in the lab: Problems and promises. Joumal of Experimental Psychology: Animal Behavior Processes, 15, 81-87.

SKINNER, B. F. (1938). The behavior of organisms: An experimental analysis. New York: Appleton-Century-Crofts.

Snapper, A. G., KAdden, R. M., a InGLus, G. B. (1982). State notation of behavioral procedures. Behavioral Research Methods \& Instrumentation, 14, 329-342.

Timberlake, W., Lucas, G. A. (1989). Behavior systems and learning: From misbehavior to general principles. In $\mathbf{S}$. Klein \& R. R. Mowrer (Eds.), Contemporary learning theories: Instrumental conditioning theory and the impact of biological constraints on learning (pp. 237-275). Hillsdale, NJ: Erlbaum.

Williams, S. B. (1938). Resistance to extinction as a function of the number of reinforcements. Joumal of Experimental Psychology, 23. 506-522.

(Manuscript received November 2. 1990; revision accepted for publication May 30, 1991.) 